\title{
ÉLÉMENTS POUR UNE BIOGRAPHIE DES TASSES À CAFÉ DANS L'EMPIRE OTTOMAN
}

n observe, ces dernières années, un intérêt croissant pour l'archéologie du monde ottoman ${ }^{1}$ et pour l'étude des artefacts trouvés en fouilles ${ }^{2}$. Parmi eux, de petits récipients, identifiés comme des tasses à café, apparus sur de nombreux sites urbains et ruraux, sont considérés par les archéologues comme des marqueurs des niveaux ottomans. Si les travaux érudits ne manquent pas sur le thème du café — son histoire, son commerce, ses lieux de consommation et les objets nécessaires à sa préparation $^{3}$ — , rares sont les auteurs qui se sont attardés sur la tasse à café,

Véronique FrançoIs est chargée de recherche au Laboratoire d'archéologie médiévale méditerranéenne, Aix-en-Provence.

vfrancois@mmsh.univ-aix.fr

1 U. Baram, L. Caroll (eds.), A Historical Archaeology of the Ottoman Empire. Breaking New Ground, New York, Kluwer Academic/Plenum Press, 2000; I. GERELYES, G. Kovacs (eds.), Archaeology of the Ottoman Period in Hungary, Budapest, 2003; F. YenişEHIRLIOĞLU, «L'archéologie historique de l'Empire ottoman. Bilan et perspectives », Turcica 37, 2005, p. 245-266.

2 M. Milwright, «Pottery of Bilad al-Sham in the Ottoman Period: a Review of the Published Archaeological Evidence», Levant 32, 2000, p. 189-208; idem, «Written Sources and the Study of Pottery in Ottoman Bilad-al-Sham » à paraître; V. FrançOIS, «Tabak, ibrik, fincan et autres pots d'époque ottomane au Bilâd al-Châm », Turcica 37, 2005, p. 281-309.

3 R.S. Hattox, Coffee and Coffehouse, The Origins of a Social Beverage in the Medieval Near East, University of Washington Press, 1988; U. BARAM, «Clay Tobacco Pipes and coffe Cup Sherds in the Archaeology of the Middle-East: Artifacts of Social Tensions from the Ottoman Past», International Journal of Historical Archaeology 3, 1999, p. 137-151 ; M. TUCHSCHERER (éd.), Le commerce du café avant l'ère des plantations coloniales. Espaces, réseaux, sociétés (XV $X V^{e}-X X^{e}$ siècle), Cahiers des annales islamologiques 20, 2001. 
à l'exception de H. Desmet-Grégoire qui lui a consacré un chapitre entier dans sa monographie sur Les objets du café examinés du point de vue de l'anthropologue 4 . Aussi complète que soit son approche, elle s'appuie sur d'abondantes sources écrites et sur les fonds des musées des arts et traditions populaires mais ne prend pas en compte la réalité matérielle livrée par le terrain archéologique. Or cette dernière est différente, voire même en opposition avec ce que les sources écrites rapportent sur la façon de boire le café.

Ce travail se propose donc d'examiner la tasse dans sa trajectoire globale, de confronter les mots aux choses, en essayant de ne pas se laisser abuser par la mise en scène des objets à travers les textes et les images. Il s'agit d'écrire une histoire de la tasse à café dans les mondes arabe et turco-ottoman en mettant en parallèle les sources écrites et les données matérielles tout en sachant «qu'un témoignage oral ou écrit peut omettre ou tromper, dissimuler ou mentir, l'objet, lui, ne ment pas $»^{5}$.

\section{LIEUX ET MODE DE CONSOMMATION DU CAFÉ À L'ÉPOQUE OTTOMANE}

La culture du café a probablement commencé dès les $\mathrm{XIII}^{\mathrm{e}}-\mathrm{XIV}^{\mathrm{e}}$ siècles sur les Hauts Plateaux d'Éthiopie mais sa consommation et sa diffusion sur une plus large échelle n'ont pas débuté avant le début du $\mathrm{XV}^{\mathrm{e}}$ siècle dans les milieux soufis du Yémen ${ }^{6}$. La consommation de café, même si elle fut un temps considérée comme illicite et interdite par les autorités religieuses et politiques, se propagea rapidement dans l'Empire ottoman. Elle est surtout balisée dans les villes. Le café est attesté à La Mecque vers 1511 puis, durant le premier quart du XVI siècle, en Egypte, chez les mystiques musulmans de l'université d'alAzhar au Caire. Dans cette ville, le goût pour cette boisson nouvelle ne cessa de progresser jusqu'à la fin du XVIII ${ }^{\mathrm{e}}$ siècle et, selon les voyageurs, hommes et femmes de presque toutes les couches sociales y

4 H. Desmet-Gregoire, Les objets du café, Paris, Presses du CNRS, 1989; eadem (dir.), Contributions au thème du et des cafés dans les sociétés du Proche-Orient, Aix-enProvence, Cahiers de l'IREMAM, 1991.

5 J. JAMIN, «Introduction à Miroirs de l'Afrique», dans M. LeIRIS, Miroirs de l'Afrique, Paris, Gallimard, 1996, p. 19.

${ }^{6}$ Durant les séances de $d h i k r$ des Soufis — une cérémonie communautaire à forte charge mystique avec un long récitatif - le café maintenait en éveil le consommateur et rendait son esprit plus vif. M. TUCHSCHERER, «Commerce et production de café en mer Rouge au XVI ${ }^{\mathrm{e}}$ siècle», dans M. TuCHSCHERER (éd.), 2001, p. 69; idem, «Café et cafés dans 1'Egypte ottomane (XVI'-XVIII' siècles)», dans H. DeSMET-GrEGOIRE, 1991, p. 56 ; E. Issin, «Coffeehouses as Places of Conversation» dans S. FAROQHI, C.K. NeumanN (eds.), The Illuminated Table, the Prosperous House, Beiruter Texte und Studien 73, Würzburg, 2003, p. 203-204, note 9. 
avaient pris goût ${ }^{7}$. Très vite, sa consommation devint alors tout à fait commune et dépassa rapidement son cadre urbain initial pour se diffuser aussi bien dans les sociétés paysannes des campagnes que parmi les bédouins du désert ${ }^{8}$. Dans la province ottomane de Châm, un document, daté de la fin de l'année 1565, fait état de l'introduction récente du café à Jérusalem et, à Damas, venu directement du Hedjaz par la caravane du Pèlerinage ${ }^{9}$, il était déjà populaire vers 1542-43. C'est également au cours de la première moitié du $\mathrm{XVI}^{\mathrm{e}}$ siècle, que cette boisson parvint jusqu'à Istanbul. Au milieu du siècle suivant, le café, qui était déjà apprécié par les classes supérieures de la société turco-ottomane et par la cour, gagna l'ensemble des classes moyennes et se répandit parmi les classes populaires.

Conséquemment à la diffusion de cette boisson «plus noire et amère que la suye détrempée ${ }^{10}$ apparurent en grand nombre des établissements destinés à sa consommation. Evliya Çelebi évaluait à 643 le nombre de cafés ouverts au Caire à la fin du XVII siècle. En 1660, le voyageur Henry Castela constatait en effet qu'en «ceste cité, il y a grand nombre de tavernes et de cabarets, où l'on vend un certain breuvage noir que les Mores boivent tout chaud. À la pointe du jour et à toute heure, il s'y en trouve quatre ou cinq cents, à la fois, qui vont boire de cette liqueur ou breuvage » ${ }^{11}$. Lors de l'expédition d'Egypte, Chabrol retint le nombre de 1200 cafés pour la ville du Caire, 100 pour le port de Bûlâq et 50 pour le Vieux Caire ${ }^{12}$. Les bayt qahwa, situés pour la plupart dans les souks, étaient des établissements de taille modeste équipés d' "une estrade en planches qui forme une espèce de sofa circulaire autour de la salle, quelques nattes de feuilles de palmier, et des tapis grossiers pour les plus somptueux, une espèce de comptoir du bois le plus ordinaire, tels sont les meubles qui se trouvent dans les cafés égyptiens ${ }^{13}$. Des documents d'archives du XVIII ${ }^{\mathrm{e}}$ siècle confirment la modestie des débits de café et la pauvreté du mobilier constitué de deux ou trois bancs en bois, de quelques meubles de rangements pour y entreposer les tasses et d'une ou deux étagères pour les ustensiles nécessaires à la préparation du breuvage ${ }^{14}$. À Damas, l'établissement le plus ancien mentionné dans les sources est le café constitué en waqf par Murad Pacha, en 1577, à

7 M. TUCHSCHERER, 1991, p. 53-73.

8 Ibidem, p. 53-54.

9 A.K. RAFEQ, «The Socioeconomic and Political Implications of the Introduction of Coffee into Syria. 16th-18th Centuries », dans M. TuCHSCHERER (éd.), 2001, p. 129-130, 133.

10 B. Monconys, Voyage en Egypte, Le Caire, IFAO, 1973, p. 56.

11 Cité par H. Desmet-Gregoire, 1989, p. 12.

12 Chabrol, Essai sur les mours des habitants modernes de l'Egypte, Description de l'Egypte, Etat moderne, vol. XVIII (1), Paris, Panckouke, 1826, p. 158.

13 Ibidem, p. 159.

14 M. TUCHSCHERER, 1991, p. 59-62, 68. 
proximité de sa mosquée dans le quartier de Suwayqa ${ }^{15}$. Les descriptions des premiers qahwa hâna dans cette ville sont peu nombreuses et font état d'espaces simplement équipés de banquettes, d'un bassin et d'un foyer. À la fin du XVII ${ }^{\mathrm{e}}$ siècle, les grands cafés installés sur les rives du Baradâ pouvaient accueillir un grand nombre de clients, de 400 à 500 personnes d'après Maundrell ${ }^{16}$. Mais les archives des tribunaux, datées du XVIII ${ }^{\mathrm{e}}$ siècle, signalent aussi l'existence d'une trentaine de ces nouveaux lieux de sociabilité masculine à l'intérieur du tissu urbain — dans les souks du centre ou dans les marchés de quartier, souvent à proximité des mosquées et des hammams, dans des secteurs particulièrement fréquentés. Ces débits de café étaient moins vastes que ceux décrits par les voyageurs et résultaient parfois de l'agencement de petites boutiques. Au début du XIX ${ }^{\mathrm{e}}$ siècle, des actes d'achat concernant le matériel des cafés de Damas indiquent que l'on trouvait dans ces établissements, des nattes, des tapis, des chaises ou des tabourets, des divans, des coffres en bois, des lampes à huile suspendues au plafond, des narguilés et quelques tasse ${ }^{17}$. Ils témoignent, comme pour Le Caire, d'un aménagement sommaire des lieux. À Istanbul, si le sultan et son entourage buvaient du café depuis 1522, les premiers kahvehâne furent ouverts par un Aleppin et un Damascène vers 1554-1555 et, dès la fin du XVI ${ }^{\mathrm{e}}$ siècle, ces établissements étaient largement répandus dans un certain nombre de villes d'Anatolie dont Bursa où Evliya Çelebi comptait 75 cafés au XVII ${ }^{\mathrm{e}}$ siècle $^{18}$. Au XVIII ${ }^{\mathrm{e}}$ siècle, cette «liqueur» faisait partie intégrante du mode de vie de tout Ottoman comme en témoigne M. d'Ohsson, le Secrétaire de sa Majesté le Roi de Suède et chargé d'affaires à la Cour de Constantinople: "Au moment du sortir du dîner on présente la pipe et le café, et c'est par où se terminent tous les repas, soit du jour, soit de la nuit. Ces usages sont presque universels. Ils s'observent dans les dernières classes des citoyens, comme chez les premiers seigneurs de la Cour ${ }^{19}$.

Ainsi la consommation de café a été presque simultanée dans les diverses provinces orientales de l'Empire ottoman. Il était servi dans des établissements qui pouvaient accueillir 200 à 400 personnes mais aussi dans de modestes boutiques équipées sommairement. Dans ces différents

15 J.-P. PASCuAL, «Café et cafés à Damas: contribution à la chronologie de leur diffusion au XVI ${ }^{\mathrm{e}}$ siècle », Berytus 42, 1995-1996, p. 151-152.

16 MAUndreLl, A Journey from Aleppo to Jerusalem at Easter 1697, Londres, 1810, p. 173.

17 B. MARINO, "Cafés et cafetiers de Damas aux XVIII ${ }^{\mathrm{e}}$ et XIX ${ }^{\mathrm{e}}$ siècles », REMM 75 76, 1995, p. 279-280.

${ }^{18}$ S. FAROQHI, Coffee and Spicies: Official Ottoman Reactions to Egyptian Trade in the Later Sixteenth Century, Wiener Zeitschrift für die Kunde des Morgenlandes, 76, 1986, p. 89; A. SARAÇGIL, «L'introduction du café à Istanbul (XVIe-XVIII' siècles)» dans H. Desmet-Gregoire, F. Georgeon (dir.), Cafés d'Orient revisités, Paris, CNRS Editions, 1997, p. 25-38.

19 M. D’Ohsson, Tableau général de l'Empire Othoman, 7 vol., Paris, 1791, p. 37-38. 
lieux, les clients buvaient le café de manière collective ou individuelle. $\mathrm{Au} \mathrm{XV}^{\mathrm{e}}$ siècle, dans les cercles soufis, une louche plongée dans un bassin de terre rempli de café passait de l'un à l'autre des mystiques ${ }^{20}$. Cette manière de boire le café en groupe, en faisant circuler un seul récipient, souvent un grand bol de céramique, paraît avoir été un temps la règle. Une interdiction officielle, établie à Damas en 1543-44, rapporte que le Grand Juge nommé par Istanbul «fait proclamer à l'intention des $d u k$ kân al-qhwa que l'on ne doit pas boire en compagnie, avec des chants et en faisant tourner des bols (al-zabâdî al-sînî) ${ }^{21}$. Cette pratique est aussi attestée, vers 1580, à Alexandrie: «Une autre boisson dénommée «Chaube» est proposée dans les tavernes; de couleur brun-noir ils s'en servent le matin. C'est à ce moment que de nombreux Turcs se rassemblent devant la taverne, souvent en groupe. Ils se laissent offrir à tour de rôle une coupe ou un bol de céramique qu'ils boivent poliment l'un après l'autre, aussi chaud qu'ils peuvent le supporter ${ }^{22}$. Le recours aux bols communs se maintiendra dans certaines régions jusqu'à une date fort avancée puisque, en 1822, les Turkmènes, vivant au nord-ouest d'Alep, buvaient leur café dans des tasses «trois fois plus grandes que celles qui sont utilisées communément dans le Levant, aussi larges que des tasses à café anglaises ${ }^{23}$. Bols et tasses de contenances différentes semblent avoir coexisté dans les débits de café comme l'observe le médecin et botaniste allemand L. Rauwulf qui voyageait en 1580 d'Alep à Bagdad: "The Arabs will drink it early in the morning, also in public places without any diffidence, from earthenware and porcelain cups. They sip it as hot as possible, and pass the cup around while sitting in a ring ${ }^{24}$. Le café était aussi servi dans des récipients individuels de très petite taille comme le rapporte d'Ohsson: «La passion des Orientaux pour cette liqueur est au-dessus de toute expression... Si la visite est longue, on en donne une seconde, même une troisième tasse, à des reprises différentes. Il est vrai que chez eux les tasses sont petites; il en faut trois ou quatre pour en faire une de celles dont on se sert en Europe $»^{25}$. Pour sa part, Ch. White s'étonne du nombre important, de 10 à 15 tasses de café consommées en un jour par une personne, mais nuance ces chiffres en précisant que toutes ces tasses réunies n'excèdent pas le contenu de deux grandes tasses de thé ${ }^{26}$. Le volume d'un fincan se

20 R.S. HatToX, 1988, p. 74.

21 J.-P. PASCUAL, 1995-1996, p. 146.

22 S. SchweIGger, Ein newe Reyssbeschreibung auss Teutschland nach Konstantinopel und Jerusalem, Nuremberg, 1608, p. 270.

23 J.L. BuRCKHARDT, Travels in Syria and the Holy Land, Londres, 1822 (rééd. 1983), p. 20.

${ }^{24}$ L. Rauwulf, Aigentliche Beschreibung der Raisse in die Morgenlander, 1582, p. 102-103.

25 D’Ohsson, Tableau général de l'Empire Othoman, 7 vol., Paris, 1791, p. 82.

26 Ch. White, Three Years in Constantinople; or, Domestic Manners of the Turks in 1844, 3 vols., Londres, 1845, p. 280. Des chiffres plus importants sont donnés par Cha- 
situe entre 50 et $100 \mathrm{ml}$, tandis que celui d'une tasse à thé de type occidental est plutôt de $170 \mathrm{ml}$. La consommation rituelle et collective à l'aide d'un grand bol et la consommation individuelle dans de très petites tasses semblent avoir coexisté et nous verrons que la production de tasses, notamment au Yémen, s'est adaptée à ces deux pratiques.

\section{DES RÉCIPIENTS POUR SERVIR CETTE BOISSON «TOUTE NOIRE, ÉPAISSE ET BOUILLANTE»}

\section{1) Les mots}

La difficulté que j'ai rencontrée d'emblée était, pour aborder un même objet - la tasse — de traiter de documents de forme et de conception différentes, rédigés en diverses provinces de l'Empire, sur une longue période, du $\mathrm{XVI}^{\mathrm{e}}$ au $\mathrm{XIX}^{\mathrm{e}}$ siècle, par des personnes occupant des fonctions différentes et pratiquant des langues variées - les listes de termes sont parfois difficiles à appréhender parce que le turc est mélangé à l'arabe et au persan. Les inventaires après décès et les inventaires domestiques étaient consignés par des juges dont les compétences en matière de vaisselle pouvaient être limitées et les termes qu'ils employaient, sans doute génériques, ne recoupaient pas forcément, d'un document à l'autre, la même réalité. Les livres de comptes et les inventaires des Cuisines impériales, les textes relatifs au Trésor du palais, les registres de ventes et de confiscations, les tarifs des prix ont souvent été écrits par des fonctionnaires et des administrateurs qui ne possédaient pas forcément le vocabulaire adéquat pour désigner les objets qu'ils devaient consigner. Cependant, ces scribes étaient capables de distinguer les tasses les unes des autres puisque une estimation de leur valeur était proposée. Les voyageurs et autres chroniqueurs occidentaux, fort prolixes pour dépeindre cette pratique caractéristique du mode de vie des Ottomans, ont pu faire des confusions lorsqu'ils ont décrit les objets qu'ils découvraient, ne maîtrisant pas toujours suffisamment la langue du pays dans lequel ils se trouvaient. Enfin, des termes identiques, selon qu'ils étaient employés à Istanbul, au Caire ou à Damas, n'avaient peutêtre pas le même sens. Cependant, de même qu'il y eut simultanéité dans l'adoption du café de l'Arabie à la Méditerranée orientale, il semble qu'il y eut aussi coïncidence dans le choix des objets servant à sa consommation. Au XVI ${ }^{\mathrm{e}}$ siècle en effet, les Ottomans étendirent leur domination sur la majeure partie du monde arabe et l'intégrèrent dans un vaste ensemble économiquement homogène ce qui réanima la circulation des personnes et des biens tout en contribuant à la diffusion d'habitudes nouvelles dont la préparation et le service du café à l'aide d'usten-

brol: «Il y a des hommes, assez pauvres d'ailleurs, qui en consomment jusqu'à trente fingân par jour », CHABROL, 1826, p. 56. 
siles assez standardisés ${ }^{27}$. Ces réserves établies, il est possible de dresser un inventaire des termes relatifs aux récipients utilisés pour boire «l'eau noire des Turcs » dans les mondes arabe oriental et turco-ottoman.

Le mot tasse (tâs), d'origine persane, désigne un récipient à boire tenu d'une seule main ${ }^{28}$ mais le terme le plus employé, dans les sources turques et arabes, pour évoquer les tasses, est fincan (du persan finjân). Ce nom est souvent accompagné d'adjectifs qui précisent sa fonction et c'est pourquoi, ayant éliminé les sakız fincan ou tasse à mastic, turşu için fincan ou coupelle pour les légumes marinés et fincan pour le salep, on se limitera aux kahve fincanlarl autrement dit tasses pour le café29. Diverses épithètes sont utilisées pour qualifier plus précisément la nature de ces ustensiles. Fağfuriyé est un terme turc qui fait référence à l'empereur de Chine et, par association, c'est le nom générique donné aux por-

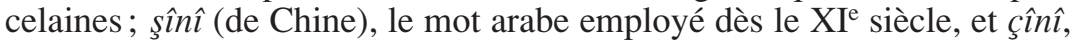
le mot persan et turc, désignent des terres cuites. Dans les documents turco-ottomans, făgfur fait référence généralement à la porcelaine et çîn à la poterie fabriquée à partir d'argile et de silice ${ }^{30}$. Le terme faïence, utilisé à de nombreuses reprises dans les traductions, est une commodité de langage et non une description de la nature de l'objet car la faïence est une terre émaillée, autrement dit une pâte argileuse couverte d'une glaçure à l'étain, qui ne correspond à aucune production de la partie orientale de l'Empire ottoman. Ainsi qu'en témoignent l'iconographie et l'archéologie, ces objets étaient de petits godets à panse hémisphérique, souvent à lèvre éversée parfois à lèvre droite, montés sur une base annulaire et plus rarement sur un piédouche (findjân muk'ab) et ne portant jamais d'anse (pl. I: 3, 4) ${ }^{31}$.

Les sources écrites permettent d'établir une liste, partielle sans doute, des types de tasses utilisés dans les grandes villes de l'Empire — dans les cafés, chez les particuliers ou à la Cour — et d'en évaluer le nombre.

En Egypte, à la fin du XVI $\mathrm{XI}^{\mathrm{e}}$ siècle, on pouvait boire le café dans des fincan en porcelaine de Chine. Un droguiste établi au Caire possédait cinq tasses de porcelaine chinoise et un marchand cairote proposait sur son étalage des tasses à café en porcelaine de Chine et en céramique $\mathrm{d}^{\prime} \mathrm{Iznik}^{32}$. Au XVIII ${ }^{\mathrm{e}}$ siècle, les fincân sînî avaient un prix élevé, de 15 à

27 Sur ce point, voir H. DESMET-GREGOIRE, 1989.

28 A.S. Melikian-ShIRVANI, Islamic Metalwork from the Iranian World 8th-18th Century, Londres, Victoria and Albert Museum Catalogue, 1982, p. 401.

29 J. Raby, Ü. YüCEL, dans R. KRAHL, Chinese Ceramics in the Topkapı Saray Museum, A Complete Catalogue, vol. I, Londres, Sotheby's Publications, 1986, p. 69.

30 J. RABY, «Fağfur, Merteban and other Terms for Porcelain and Celadon», dans R. KRAHL, 1986, p. 82.

31 N. de Blegny, Le bon usage du thé, du caffé et du chocolat pour la preservation \& pour la guerison des maladies, Lyon, Amaulry, 1687, p. 168.

32 C'était une marchandise importante dans le commerce d'importation à partir des ports de la mer Rouge comme en attestent les multiples inventaires après décès de négo- 
20 paras, et c'est sans doute pourquoi leur propriétaire les faisait réparer par des artisans spécialisés ${ }^{33}$. Dans un café installé près du Khân el-Khalili, 13 tasses en bon état côtoyaient 23 fincân madjbûr, c'est-à-dire des tasses réparées ${ }^{34}$. Au XIX ${ }^{\mathrm{e}}$ siècle, les buveurs de café utilisaient encore des porcelaines chinoises comme en atteste E.W. Lane «The coffeecup... is of porcelain, or Dutch-ware and being without a handle ${ }^{35}$ mais également des tasses importées d'Europe. Selon Chabrol, les tasses «sont quelque fois en porcelaine, et viennent d'Allemagne ${ }^{36}$. Dans les tavernes du Caire, d'autres récipients, plus rustiques, arrivaient du Yémen - un cafetier, au début du XVII ${ }^{\mathrm{e}}$ siècle, possédaient 60 tasses importées de la ville de Hays pour une valeur totale de 70 paras, soit 0,8 paras pièce ${ }^{37}$. Mais le café n'était pas uniquement servi dans des objets importés, on le buvait aussi dans de petites tasses «blanches ou à fleurs, et qu'on appelle fingân belady par opposition à celles qu'on apporte d'Europe » car, selon la Description de l'Egypte, les potiers cairotes exécutaient «une sorte de faïence grossière dont on fait des pots à confitures, ou des tasses à café. Au surplus, cette industrie ne mérite guère d'être citée, tant à cause de son imperfection que par la petite quantité de ces produits ${ }^{38}$. Ces objets apparaissaient parfois en grand nombre. Les inventaires après décès de notables font mention de plusieurs centaines de tasses à café d'origines variées - ainsi parmi les biens d'un émir du Caire, mort en 1631, 25 variétés de tasses à café sont décrites et évaluées et, dans l'héritage d'un notable impliqué dans le commerce de mer Rouge, 700 tasses chinoises sont recensées. Au milieu du XVII siècle, ce type d'objets faisait habituellement partie du trousseau de la mariée - une jeune épouse avait reçu en dot 180 tasses $^{39}$. Comme l'a montré M. Tuchscherer, dans les quelques établissements cairotes pour lesquels nous disposons d'inventaires pour le XVIII ${ }^{\mathrm{e}}$ siècle, le nombre de tasses variait entre 30 et 210 et se situait, en moyenne, autour de 90.

Dans les inventaires mobiliers de Damas, au XVIII ${ }^{\mathrm{e}}$ siècle, les fincan sont fréquemment décrits mais, si ces textes précisent la qualité des tasses, les termes employés recouvrent une réalité parfois difficile à

ciants cairotes de la fin du XVII ${ }^{\mathrm{e}}$ et de la première moitié du XVIII ${ }^{\mathrm{e}}$ siècle. M. TuCHSCHERER, 1991, p. 64.

33 J. von Hammer (ed.), Narrative of Travels in Europe, Asia, and Africa in the Seventeenth Century by Evliya Effendi, Londres, 1834, vol. I, part II, p. 212.

34 M. TuCHSCHERER, 1991, p. 64.

35 Dutch Ware fait référence aux porcelaines importées de Chine par la Dutch East India Company. E. W. LANE, Manners and Customs of the Modern Egyptians, Londres, East-West Publications, rééd. 1981, p. 137.

36 ChaBrol, 1826, p. 159.

37 M. TuCHSCHERER, 2001, p. 88.

38 Description de l'Egypte. Etat Moderne, t. XII, p. 473 et t. XVII, p. 205, Paris, Panckouke, 1824.

39 N. Hanna, «Coffee and Coffee Merchants in Cairo 1580-1630», dans M. TuCHSCHERER (éd.), 2001, p. 97-98. 
appréhender ${ }^{40}$. On y distingue par volume décroissant de simples fincan sans qualificatif particulier, viennent ensuite des tasses 'asali, une épithète mystérieuse qui, évoquant peut-être le miel, ferait référence à la couleur du matériau ou de la glaçure; des tasses 'adjami (peut-être de Perse); des tasses sini ; des tasses qualifiées de djadid (neuves); ou encore des tasses gümüşlü, autrement dit en argent, à moins que ce terme ne fasse plutôt référence à un décor de lustre métallique ou n'évoque les sertissages en métal destinés à protéger des chocs les parties sensibles de l'objet; quelques fincan sont dits qaşani, c'est-à-dire à la manière des productions perses de Kashan et enfin les tasses fağfuri renvoient aux porcelaines de Chine. Une pièce qualifiée de may est sans doute de couleur bleue tandis que 17 autres sont blanches. Les inventaires après décès des pèlerins mort à Damas au $\mathrm{XVIII}^{\mathrm{e}}$ siècle montrent que, lors du voyage aller vers La Mecque, quatre voyageurs circulaient avec, dans leur baluchon, 47 tasses. Au retour, non seulement ils étaient plus nombreux à posséder de tels récipients, mais ces derniers étaient beaucoup plus abondants et d'une valeur inférieure à ceux emportés à l'aller dix-neuf pèlerins détenaient 412 tasses $^{41}$. Les plus onéreuses d'entre elles étaient les tasses gümüşlü d'un coût de 3,5 piastres, venaient ensuite les tasses bleues (?) à 0,5 piastres, les neuves (djadid) à 0,37 piastres, les tasses chinoises (fağfuri) à 0,14 piastres, les tasses sini à 0,11 piastres, les tasses simples à 0,10 piastres et enfin les moins chères étaient les tasses 'adjami à 0,09 et asali à 0,07 piastres.

En Anatolie aussi, les porcelaines de Chine étaient utilisées, dans certains milieux, pour boire le café. Au XVII ${ }^{\mathrm{e}}$ siècle, le stock de porcelaines chinoises du marchand Haci Yusuf d'Ankara consistait presque exclusivement en fincan. Il possédait également 5 Kabe fincani — autrement dit tasse à café de la Ka'ba — d'une valeur de 0,2 esedi guruş chacune; 22 tasses fağfuri, au prix de 0,1 esedi guruş l'une, et une tasse qualifiée de acemi beaucoup plus chère (1 esedi guruss $)^{42}$. Vers 1700, un des grands marchands de tissus de Bursa, de retour du Pèlerinage vers les Lieux Saints, rapportait, selon un inventaire établi à Damas, 137 tasses dont 70 asali, 45 de Chine - il n'était pas rare que les pèlerins achètent, à La Mecque, quelques tasses en porcelaine de Chine comme objets-souvenirs - et 27 tasses neuves (djadid) ${ }^{43}$. Pour leur part, les tarifs de prix fixes, les inventaires de succession et les registres du palais impérial à

40 C. Establet, J.P. PAscual, Ultime voyage pour La Mecque. Les inventaires après décès de pèlerins morts à Damas vers 1700, Damas, 1998, p. 87; idem, «Cups, plates, and kitchenware in late seventeenth-and-early eighteenth-century Damascus », in S. FAroqhi, C.K. Neumann (eds.), The Illuminated Table, the Prosperous House, Beiruter Texte und Studien 73, Würzburg, 2003, p. 185-198.

41 C. Establet, J.P. Pascual, 1998, p. 150-151.

42 S. FAroqhi, Men of Modest Substance. House Owners and House Property in Seventeenth-Century Ankara and Kayseri, Cambridge University Press, 1987, p. 28.

43 C. Establet, J.P. Pascual, «Café et objets du café dans les inventaires de pèlerins musulmans vers $1700 »$, dans M. TUCHSCHERER (éd.), 2001, p. 149. 
Istanbul, datés des $\mathrm{XVI}^{\mathrm{e}}$ et début $\mathrm{XVII}^{\mathrm{e}}$ siècles, ne sont pas avares de détails sur les récipients destinés à boire le café puisqu'on y recense des tasses multicolores, des tasses vertes, des tasses persanes, des tasses piriformes, des tasses «rossignols», des tasses güllü paşa et leurs soucoupes, des tasses bleues à bec, des tasses à pied ${ }^{44}$. Certains récipients sont qualifiés de paşa fincan, mais il est difficile de savoir si les objets ainsi désignés étaient simplement plus grands que la norme ou s'ils étaient destinés à des personnages de haut rang et donc de qualité supérieure. Dans le Tarif des prix fixes (narh defterleri) du 15 septembre 1600 , des tasses à café (kahve fincalarl) ont un prix qui varie du simple au double selon qu'elles sont simples (sade) ou dorées (altunli) (2 akçe contre 5). Un second Tarif, daté du 31 décembre 1640, fait état, parmi la vaisselle d'Iznik, de tasses à motifs (alaca) et de tasses vertes. L'inventaire de la succession de Bostancıbaşı Süleyman Ağa, daté de 1605, témoigne de sa richesse notamment parce qu'il possédait 43 bols (kâse), plats (tabak) et tasses (fincan) d'Iznik et 15 pièces de porcelaines chinoises. Un inventaire des cuisines impériales, daté de 1617-18, recense 401 fincan et 60 petites tasses au prix de 2 akçe la pièce, tandis que dans un registre de comptes de 1631, on trouve 203 fincan à 3,39 akçe. Toutes ces données concernent le palais, les hauts dignitaires et les riches commerçants mais les inventaires mobiliers des cafés font défaut. Nous ne savons donc pas dans quel type de récipient les gens du peuple buvaient leur café, sinon qu'au XIX ${ }^{\mathrm{e}}$ siècle, dans les rues d'Istanbul, des marchands ambulants servaient ce breuvage dans des gobelets de métal ${ }^{45}$ (pl. V: 1). Il semble qu'au Yémen, à la fin du XVIII ${ }^{e}$ siècle, tous ces objets coexistaient. En effet, selon Carsten Niebuhr «Les Arabes distingués du Yémen ont des tasses de la Chine et quelquefois des soucoupes pareilles, mais plus ordinairement, leurs soucoupes, comme chez les Arabes septentrionaux et chez les Turcs, sont d'argent ou de laiton. Le peuple a des tasses de pierre de potier ${ }^{46}$ (pl. I : 5).

\section{2) Les choses}

Les sources écrites font état de plusieurs catégories de tasses qui peuvent être identifiées, à partir des descriptions les plus claires, avec des porcelaines de Chine, des céramiques anatoliennes d'Iznik, des porcelaines importées d'Allemagne et des terres cuites venues du Yémen. Pour leur part, les qualificatifs de çînî, belady, qaşani et 'ajami permettent, sinon de définir l'origine des objets, du moins d'en approcher la nature. Grâce aux artefacts trouvés en fouille ou conservés dans les

44 N. Atasoy, J. Raby, Iznik, La poterie en Turquie ottomane, Ed. du Chêne, 1990, p. 23-27, 30, 31, 47.

45 J. B. van Moor, Recueil de cent estampes representant differentes nations du Levant tirees sur les tableaux peints d'apres nature en 1707. et 1708. par les ordres de m. de Ferriol, ambassadeur du roi a la Porte, Paris, Le Hay, 1714, pl. 56.

46 C. Niebuhr, Description de l'Arabie, Paris, Brunet, 1779, p. 80. 
musées, il est possible de donner une réalité matérielle aux désignations employées dans les textes. Par ailleurs, un examen des découvertes faites sur divers sites archéologiques disséminés dans l'Empire précisera la diffusion de ces objets, telle qu'elle est révélée par le terrain et non plus à partir des documents d'archive, et les contextes dans lesquels ils furent utilisés.

\section{Tentative d'identification des types de tasse évoqués dans les textes}

\section{- Les tasses fağfuri}

Comme le révèlent les textes et ainsi que le confirme l'archéologie, une partie des tasses employée dans l'Empire ottoman pour boire le café était des gobelets en porcelaine de Chine dont s'inspirèrent par la suite les manufactures d'Iznik et de Kütahya pour réaliser des objets de même forme et parfois de même style décoratif.

Les tasses de porcelaine, fabriquées en Chine, s'exportèrent en grand nombre à partir du $\mathrm{IX}^{\mathrm{e}}$ siècle $^{47}$. Cependant, nous ignorons à quel usage étaient destinées ces petites coupes, importées, au Proche et au Moyen Orient, bien avant l'arrivée du café dans ces régions. Et nous ne connaissons pas les raisons qui poussèrent Arabes et Turcs à adopter ces récipients chinois pour boire le café, des « objets dépaysés », selon l'expression de H. Desmet-Grégoire, passés d'une culture à une autre et adaptés à de nouvelles pratiques. En effet, les gobelets de porcelaine servaient en Chine à la consommation de boissons diverses : du vin, de l'alcool de riz ou du thé ${ }^{48}$. Dans l'Empire du Milieu, avant l'apparition des premiers ustensiles en porcelaine, sous la dynastie des T'ang (618-907), les Chinois buvaient le thé depuis plusieurs siècles «à la manière d'un bœuf assoiffé » dans de rustiques grands bols en bois ${ }^{49}$. Mais, sous la dynastie des Song (960-1279), le service du thé devint une pratique raffinée du quotidien. La porcelaine prit alors une place déterminante dans l'univers qui entourait le thé. L'utilisation de petites tasses en porcelaine - un matériau qui avait la propriété de garder la chaleur du liquide — impliquait non seulement de déguster le thé avec plus de parcimonie, mais aussi de veiller à la qualité d'un breuvage que cette matière si pure ne pouvait altérer et exaltait. Le choix délibéré de certaines tasses pour les couleurs qu'elles pouvaient ajouter au breuvage montre bien l'impor-

47 Des tessons de céladons de Yüeh ont effectivement été mis au jour à Samarra et à Fostat pour l'époque T'ang et des tasses sont aussi mentionnées dans la liste des présents que le gouverneur du Khorassan offrit au sultan Al-Rashid, qui gouverna à Bagdad de 786 à 809. T. Mikami, «Chinese Ceramics from Medieval Sites in Egypt», in Prince T. Mikasa (ed.), Bulletin of the Middle Eastern Culture Center in Japan, vol. II : Cultural and Economic Relations between East and West Sea Routes, Wiesbaden, 1988, p. 10 ; Y. CROwE, «Early Islamic Pottery and China», TOCS 1977, p. 263-264.

48 H. Desmet-Gregoire, 1989, p. 76.

49 K. Cha Sangmanee, C. Donzel, St. Melchior-Durand, A. Stella, L'ABCdaire du thé, Flammarion, 1996, p. 92. 
tance des contenants: «The best tea cups are made of Yüeh and of Hsing porcelain. Yüeh is blue-green, it is like jade, the tea becomes green in it. Hsing is like silver, white, is inferior, and the tea becomes red in it ${ }^{50}$. Les récipients employés pour servir le thé étaient alors des bols cylindriques, tronconiques ou hémisphériques, de grès ou de porcelaine, d'un diamètre variant entre 11 et $12 \mathrm{~cm}$ pour une hauteur de 7 à 10 $\mathrm{cm}$. Les bols à thé Jian, fabriqués dans la province du Fujian, étaient très réputés à l'époque des $\operatorname{Song}^{51}$ (pl. II : 1 et 2 ) ainsi que les céladons de Hangzou et de Lonqguan (pl. II : 3-5). Sous les Ming (1368-1644), le thé se démocratisa et gagna l'ensemble des classes sociales, il était alors servi dans des tasses de «bleu et blanc» (pl. II : 6 et 7).

Selon les découvertes archéologiques, les tasses chinoises utilisées dans l'Empire, à partir du XVI $\mathrm{XV}^{\mathrm{e}}$ siècle, pour boire le café étaient des «bleu et blanc», c'est-à-dire des porcelaines peintes au bleu de cobalt, ornées de décors végétaux stylisés - des fleurs de lotus ou de chrysanthèmes, des lingzhi ou champignons sacrés, des fleurons divers - , de paysages dans lesquels évoluent parfois des animaux - des oiseaux perchés sur des rochers, des échassiers, des dragons au milieu de nuages ou sortant des flots écumants — ou de quelques pêcheurs (pl. II : 6-8). Ces vases étaient fabriqués dans les ateliers chinois à la fin de l'époque Ming et, pour la plus grande part, à l'époque dite de «Transition » puis sous la dynastie Qing, c'est-à-dire aux XVII et XVIII ${ }^{\mathrm{e}}$ siècles. D'autres tasses en porcelaine, du XVIII ${ }^{\mathrm{e}}$ siècle, dites «café-au-lait» ou Deaf-Leaf Brown, se distinguent du groupe précédent par une couverte allant du jaune miel au chocolat employée seule ou parfois associée à des décors peints en bleu (pl. II : 9). Il est possible que cette couverte corresponde au qualificatif 'asali (en rapport avec le miel ?) qu'on rencontre dans les inventaires damascènes du XVIII ${ }^{e}$ siècle. Plus rares, certaines tasses, datées du $\mathrm{XIX}^{\mathrm{e}}$ siècle, sont entièrement couvertes, à l'extérieur, d'un bleu de cobalt profond, dit bleu poudré, servant de fond à un décor peint en doré. Enfin, les tasses à café ne sont pas rares parmi les imitations chinoises des imari japonais qui combinent le bleu et le rouge sous couverte à des rehauts d'or, une production devenue, peu après son introduction au début du XVIII ${ }^{\mathrm{e}}$ siècle, une marchandise d'exportation très populaire (pl. II : 10). Ces tasses venues de Chine ont très rapidement été adoptées pour servir le café, sans doute choisies parce que la porcelaine, dure, fine et légère gardait la chaleur du liquide. Elle était donc bien adaptée pour le café dont la température devait être élevée au moment de

\footnotetext{
50 C'est un passage du premier traité sur le thé, le Ch'a Ching, composé par Lu Yü (723-804) et repris par Chu Yen dans le T'ao Shuo — une compilation sur la céramique chinoise écrite en 1774 à partir de sources anciennes. P. KAHLE, "Chinese Porcelain in the Lands of Islam », TOCS 1940-14, p. 36.

51 J. Ayers, H. Fromentin, M. Paul-David, A. TAmburello, La céramique d'Extrême-Orient, Paris, 1984, p. 112, pl. 84.
} 
sa dégustation car «ce breuvage se prend à petites gorgées le plus chaud que faire se peut $\gg^{52}$. Il y eut adéquation presque parfaite de la forme et de l'usage puisque les principaux ateliers ottomans reprirent les formes et parfois les décors de ces petits objets chinois pour créer leurs propres tasses à café qui cependant n'étaient pas des porcelaines. Le petit gobelet à panse hémisphérique monté sur une petite base annulaire devint alors le récipient type pour déguster cette «liqueur toute noire, épaisse et bouillante». La tasse à café était née. Et de même qu'aujourd'hui une bolée de cidre a moins de saveur si elle est prise dans un autre contenant ou que le vin servi dans un verre épais est moins goûteux, le service du café nécessitait, dans les mondes arabe et turco-ottoman, le fincan.

\section{— Les tasses d'Iznik et de Kütahya}

Parmi la grande variété d'objets produite dans les ateliers d'Iznik, les fincan tenaient leur rang comme en attestent les documents d'archives. Mais dans les fouilles archéologiques, alors que la vaisselle d'Iznik n'est pas rare, les tasses à café sont exceptionnelles. Les pièces entières sont présentées aux musées d'Iznik, d'Istanbul et de Bursa (pl. III : 1, 2). Ces tasses étaient fabriquées dans une pâte siliceuse très dure, à fritte plombifère, recouverte d'une fine couche d'engobe blanc siliceux sur laquelle les décors étaient peints à l'aide d'oxydes de cobalt pour le bleu, d'oxydes de manganèse pour le violet, d'oxydes de chrome pour le vert olive, d'oxydes de cuivre pour le vert émeraude et de sable ferrugineux pour le rouge. L'ensemble était revêtu d'une glaçure transparente, alcalino-plombifère, mélangée à une solution d'étain ${ }^{53}$. À côté de ces objets polychromes, des tasses à glaçure monochrome verte ont aussi été fabriquées à Iznik ${ }^{54}$. À l'inverse, les fincan de Kütahya constituent presque une constante des niveaux d'occupation d'époque ottomane. Kütahya, en Anatolie centrale, était célèbre pour ses productions de vaisselle dès le $\mathrm{XIV}^{\mathrm{e}}$ siècle, mais c'est à partir du XVIII ${ }^{\mathrm{e}}$ que la production de cette ville se distingue par la fabrication de pièces de formes et de carreaux de revêtement à pâte alcalino-calcaire, peints au bleu de cobalt sur fond blanc ou à l'aide d'une palette colorée particulièrement riche constituée d'un jaune citron très lumineux, d'un bleu de cobalt, d'un turquoise et d'un vert émeraude de cuivre, d'un violet de manganèse et d'un sable ferrugineux pour le rouge — appliqués sous une glaçure alcalino-plom-

52 D'après Wojcied Bobowski, plus connu, à la fin du XVII ${ }^{\mathrm{e}}$ siècle, sous le nom d'Ali Ufki Bey cité par A. ERDOĞDU, «Les repas et l'art culinaire» dans Topkapi à Versailles, Trésors de la cour ottomane, Musée national des châteaux de Versailles et de Trianon, 4 mai-15 août 1999, Paris, 1999, p. 146-147.

53 M. TITE, «The Technological Relationship between Islamic and Chinese Glazed Ceramics prior to the 16th Century AD», dans les extraits du colloque de la Société française d'étude de la céramique orientale, Chine-Méditerranée, Routes et échanges de la céramique avant le XVI siècle, Paris, musée Guimet, 26-27 novembre 2004, p. 36.

54 O. Aslanapa, Ş. Yetkin, A. Altun, The Iznik Tile Kiln Excavations (The Second Round: 1981-1988), Istanbul, 1989, p. 86, 144, 179. 
bifère transparente ${ }^{55}$ (Pl. III : 3-9). Les fincan produits dans ces ateliers, parfois accompagnés d'une soucoupe, étaient très répandus dans l'Empire et même hors de ses frontières — en 1753, par exemple, le consul de France en Crimée précisait que s'y vendaient chaque année «deux cents paniers de fayences de Cutahié de toute espèce, comme pots, vases de toutes grandeurs, tasses à sorbet et à café » ${ }^{56}$.

— Les tasses en porcelaine de Saxe

Dès le deuxième quart du $\mathrm{XVIII}^{\mathrm{e}}$ siècle, les porcelaines européennes de Meissen et de Vienne, groupées sous le nom générique ottoman de saksunya, étaient très appréciées dans l'ensemble de l'Empire, et de nombreuses tasses y étaient importées. En 1732, un négociant turc passa commande, à Meissen, de 2000 tasses à café de type fincan, aussi nommées Turkencöpgen en Saxe. Ce même marchand, en 1734, signa avec la Manufacture un contrat aux termes duquel il s'engageait à acheter tous les ans la même quantité de tasses ${ }^{57}$. Ces pièces étaient exécutées à la commande dans le goût des clients turcs qui fournissaient des modèles et imposaient un type particulier de décors adaptés au goût ottoman les fabricants allant même jusqu'à remplacer les épées croisées, une des marques de fabrique de Meissen, par un croissant ou par un caducée, parce qu'elles rappelaient trop la croix chrétienne aux clients musulmans. Les tasses de Meissen, des coupelles sans anse réalisées en porcelaine, étaient ornées de décors floraux stylisés souvent peints en polychromie, parfois rehaussés de filets dorés (pl. III : 10, 11).

- Les tasses çînî, qaşani, belady et 'ajami

Il est difficile de préciser l'exacte origine de ces objets, cependant leur nature peut être définie grâce au matériel trouvé en fouilles. Il est vraisemblable que çînî, qaşani — en référence aux fabrications perses de Kashan - et belady renvoient à un même type de production, c'est-àdire des tasses à pâte siliceuse, peintes polychromes, sous glaçure alcaline incolore ou, plus simplement, couvertes d'une glaçure monochrome bleu de cobalt, violet de manganèse ou verte. Les découvertes damascènes - des tasses à pâte siliceuse peintes au bleu de cobalt sous une glaçure alcaline incolore souvent marquées, sous la base, d'une spirale inscrite dans un carré (pl. IV : 1-4) — illustrent cette catégorie. Il est malaisé de trouver une équivalence matérielle aux tasses qualifiées

55 Ş. Akalin, H. Yilmaz Bilgi, Delights of Kütahya, Kütahya Tiles and Pottery in the Suna \& Inan Kıraç Collection, Istanbul, 1997, p. 9; J. CARswell, C.J.F. DowseTt, Kütahya Tiles and Pottery from the Armenian Cathedral of St. James, Jérusalem, vol. I, 1972; J. Carswell, Kütahya Tiles and Pottery from the Armenian Cathedral of St. James, Jérusalem, vol. II, 1972; L. Soustiel, Splendeurs de la céramique ottomane du XVIe au XIX ${ }^{e}$ siècle, Istanbul, 2000, p. 104-108

${ }^{56}$ Cité dans J. Carswell, 1972, p. 16.

57 J. Soustiel, La céramique islamique, Fribourg, 1985, p. 348-350. 
d' 'ajami, un terme qui signifie «étranger» ou «de Perse ». La dernière acception pourrait renvoyer aux tasses iraniennes à pâte siliceuse et glaçure alcaline, peut-être peintes au lustre métallique, d'époque safavide (pl. IV : 5).

\section{- Les tasses simples}

Ce sont sans aucun doute les tasses les plus difficiles à identifier puisque aucune épithète ne permet d'approcher leur origine ou leur nature. C. Niebuhr évoque, à la fin du XVIII ${ }^{\mathrm{e}}$ siècle, «des tasses de pierre de potier» utilisées par le peuple au Yémen (pl. I: 5). Si on ne connaît rien de cette production tardive, on en sait davantage sur les fabrications des ateliers de la ville de Hays, proche de Zabid, sur la plaine côtière de Tihâma, exportées au début du XVII ${ }^{\mathrm{e}}$ siècle, au Caire ${ }^{58}$. Les tasses issues de ces ateliers ont été étudiées par E. J. Keall qui a montré que l'évolution des productions était liée, selon toute vraisemblance, à une modification du mode de consommation du cafée ${ }^{59}$. Ainsi, au $\mathrm{XV}^{\mathrm{e}}$ siècle, les potiers de Hays fabriquaient des coupelles grossières à pâte rouge sans couverte qui étaient utilisées pour boire le café à petites gorgées, en commun, comme dans les cercles confrériques. Mais lorsque la consommation du café passa du monde des mystiques à celui des hommes ordinaires, la taille des récipients changea. Des tasses à thé chinoises en porcelaine - dont quelques fragments, notamment des bols «bleu et blanc» Ming, ont été retrouvés sur le site — ont sans doute contribué à la réduction de la taille des gobelets et à leur nouvelle ornementation. Les fouilles ont livré, pour les $\mathrm{XV}^{\mathrm{e}}-\mathrm{XVI}^{\mathrm{e}}$ siècles, de nouvelles tasses réalisées avec une argile blanche des environs - une pâte kaolinitique qui n'a pas été cuite à une température suffisamment élevée pour produire des porcelaines alors même que le matériau le permettait -, peintes en bleu et couvertes de glaçure plombifère (pl. IV: 6-9) et des petits récipients simplement glaçurés (pl. IV : 10-18).

\section{L'objet comme trace}

Les découvertes archéologiques font état, dans diverses provinces de l'Empire, de la présence de fincan. Mais aucune fouille, à ce jour, n'a été entreprise dans un qahwa hâna ou un kahvehâne livrant le contrepoint matériel des indications fournies par les textes. L'examen d'un certain nombre de trouvailles provenant de bâtiments militaires, de sites urbains et d'habitations rurales permet de dresser un panorama de la diffusion et de la fréquence d'apparition de ces objets ainsi que du volume des découvertes.

Les fouilles récentes, ouvertes dans la citadelle de Damas, ont livré un bel échantillonnage des principales catégories de tasses à café en usage

${ }^{58}$ M. TUCHSCHERER, 2001, p. 88.

59 E.J. Keall, «The Evolution of the First Coffee Cups in Yemen», dans M. TuchSCHERER (éd.), 2001, p. 35-50. 
à l'époque ottomane dans une grande ville arabe de l'Empire. Des documents juridiques, datés entre 1746 et 1791, révèlent que plusieurs cafés étaient installés dans l'enceinte même de la citadelle, l'un d'entre eux étant localisé à la porte occidentale ${ }^{60}$. Les gobelets mis au jour dans cette ville-garnison, où les janissaires impériaux vivaient avec leur famille, sont peut-être représentatifs de ce qui circulait en Syrie à cette époque ${ }^{61}$. Les objets, qui peuvent être considérés comme les premières tasses à café, sont des porcelaines chinoises «bleu et blanc» de la dynastie Ming, datées du XVI ${ }^{\mathrm{e}}$ siècle (pl. II : 6, 7). La période de «Transition » a aussi livré quelques tasses qui côtoient, au XVII ${ }^{\mathrm{e}}$ siècle, un unique fincan venu d'Iran safavide. Il est réalisé en pâte siliceuse très dure, et orné, à l'intérieur, d'un bouquet peint au lustre jaune-orangé sur une glaçure stannifère et, à l'extérieur, d'un décor floral avec des grenades peint en bleu de cobalt et lustre jaune d'or et rouge cuivre (pl. IV : 5). Il s'agit peut-être d'un fincan 'ajami. C'est le seul exemple reconnu sur les sites archéologiques du Proche-Orient. Au XVIII ${ }^{\mathrm{e}}$ siècle, la variété des tasses est plus importante, les fincan fağfuri «bleu et blanc» sont plus nombreux et sont associés aux porcelaines «Café-au-lait» et aux dérivés chinois des imari japonais (pl. II : 8-10). Pour la même époque, les tasses de Kütahya apparaissent en grand nombre (46 pièces) (pl. III : 6-9). Elles sont particulièrement bien diffusées dans l'ensemble de l'Empire ottoman mais pas de manière massive ${ }^{62}$. Au Proche-Orient, ces tasses ont été principalement retrouvées à Antioche, à Hama, à Acre, à la citadelle de Jérusalem, à la cathédrale arménienne de St James, au château de Belmont (région ouest de Jérusalem), à Burj al-Ahmar et à Zi'rin ${ }^{63}$. Enfin, quatre tasses de porcelaine de Meissen, fort endommagées, ont aussi été

${ }^{60}$ Communication personnelle de B. Marino (CNRS-IREMAM) que je remercie pour cette information.

61 B. MARINo, Le faubourg du Mĩdān à Damas à l'époque ottomane. Espace urbain, Société et Habitat (1742-1830), Damas, 1997; C. Establet, J.-P. PAscual, Familles et fortunes à Damas. 450 foyers damascains en 1700, Damas, 1994, p. 81, 155 ; A.K. RAFEQ, «Economic Relations between Damascus and the Dependant Countryside, 174371 », in A.L. Udovitch (ed.), The Islamic Middle East, 700-1900: Studies in Economic and Social History, Princeton New Jersey, 1981, p. 657.

62 Pour les sites de Grèce et de Turquie, voir: V. FrançOIs, «Eléments pour l'histoire ottomane d'Aphrodisias: la vaisselle de terre », Anatolia Antiqua IX, 2001, p. 184, 187.

63 F. WAAGÉ, Antioch-on-the Orontes. IV Ceramics and Islamic Coins, Princeton, 1948, fig. 87: 6, 7; Hama. Fouilles et recherches 1931-1938. Les verreries et poteries médiévales, Copenhague, 1957, p. 131, n 404; G. EDELSTEIN, M. Avissar, «A Sounding in Old Acre», 'Atiqot XXXI, 1997, p. 133, pl. III : 2, 3; C. Johns, «The Citadel Jerusalem. A Summary Work since $1934 », Q D A P$ XIV, 1950, p. 190, pl. LXIII : 8, 9; R.P. HARPER, D. Pringle, et al., Belmont Castle: the Excavation of a Crusader Stronghold in the Kingdom of Jerusalem, British Academy Monographs in Archaeology, $\mathrm{n}^{\circ} 10$, The British Academy, Londres, 2000, p. 114-116; D. PRINGLE, The Red Tower (al-Burj al-Ahmar) Settlement in the Plain of Sharon at Time of the Crusaders and Mamluks A.D. 1099-1516, Londres, 1986, p. 157-158, fig. 51, no 88-90; A.D. GREY, « The Pottery of the Later Periods from Tel Jezreel : an Interim Report », Levant 26, 1994, p. 60 
découvertes à la citadelle tandis qu'elles apparaissent occasionnellement dans les fouilles de Tripoli, d'Acre et de Jérusalem ${ }^{64}$ (pl. III : 10, 11). Les ateliers damascènes n'étaient pas en reste et proposèrent à la clientèle des petites tasses à pâte siliceuse, peintes au bleu de cobalt sous une glaçure alcaline incolore, souvent marquées, sous la base, d'une spirale inscrite dans un carré (pl. IV: 1-4).

En Turquie, les fouilles sont plus avares en objets du café. À Saraçhane Camii à Istanbul, beaucoup de céramiques d'époque ottomane ont été mises au jour dans des puits et des dépôts clos et, parmi elles, des tasses à café. Mais si les productions d'Iznik sont bien représentées, on ne trouve aucune trace de petites formes ayant pu servir pour le café. En revanche, les gobelets de porcelaines chinoises, datés entre la fin $\mathrm{XVI}^{\mathrm{e}}$ $\mathrm{XVII}^{\mathrm{e}}$ et le XVIII ${ }^{\mathrm{e}}$ siècle, étaient sans doute destinés à cet usage ${ }^{65}$. Ils étaient associés, sur le site, aux inévitables tasses de Kütahya et aux porcelaines de Meissen ${ }^{66}$.

À la lecture de ce qui précède, il ne faudrait pas croire que ces objets étaient réservés aux centres urbains. Des fouilles et des prospections ont aussi révélé leur présence dans des contextes ruraux. À Chypre par exemple, sur le site de Kouklia, un village à vocation agricole, des tasses d'Iznik, des «bleu et blanc» chinois de la période de «Transition» et des tasses de Kütahya étaient utilisées pour boire le café ${ }^{67}$. À Aphrodisias, en Carie, un site fameux de l'Antiquité gréco-romaine, devenu à l'époque ottomane un village connu sous le nom de Geyre et composé, au milieu du XIX ${ }^{\mathrm{e}}$ siècle, «de quarante cabanes, bâties parmi les ruines de l'antique Aphrodisias » ${ }^{68}$, les fouilles n'ont livré aucune ruine ottomane mais ont mis au jour une belle collection de vaisselle - un peu plus de 200 pièces — probablement fabriquée localement ou régionalement ${ }^{69}$. Des fincan de toutes natures faisaient partie de ces découvertes: deux tasses d'Iznik, l'une à couverte monochrome verte et l'autre à panse godronnée vert-bleu; deux tasses à pâte siliceuse à glaçure bleu turquoise opaque et à glaçure bleu nuit translucide d'origine indéterminée; trois fincan de Kütahya et une tasse de Meissen ${ }^{70}$. Sur le site de Sardes, l'antique et prospère capitale du royaume de Lydie devenue au

64 H. SAlame-SARKIS, Contribution à l'histoire de Tripoli et de sa région à l'époque des Croisades: problèmes d'histoire, d'architecture et de céramique, BAH 106, Paris, 1980, p. 226; G. Edelstein, M. Avissar, 1997, p. 133, pl. IV: 5a, b; J. Carswell, C.J.F. DowsETT, vol. I, 1972, fig. 32.

65 J.W. HAYes, Excavations at Saraçane in Istanbul, volume II : the Pottery, Princeton, 1992, p. 261-263, pl. 40-41.

${ }^{66}$ Ibidem, p. 266-268, pl. 43 ; p. 342, pl. 44 : b-d.

67 M.-L. von WartburG, «Types of Imported Table Ware at Kouklia in the Ottoman Period », RDAC 2001, p. 367, 382.

68 B. Poujoulat, Voyage dans l'Asie Mineure, Paris, 1840, p. 71; Ch. Texier, Description de l'Asie Mineure, III, Paris, 1849, p. 163.

69 V. FrançOIS, 2001, p. 147-190.

70 Ibidem, p. 183-185. 
$\mathrm{XVII}^{\mathrm{e}}$ siècle une petite ville assez florissante avec un marché hebdomadaire et un khan qui accueillait les voyageurs empruntant la Route de la Soie $^{71}$, puis décrite au milieu du XVIII ${ }^{\mathrm{e}}$ siècle comme un village bâti de maisons de pisé et habité par des paysans et des bergers semi-nomades ${ }^{72}$, les fouilles ont mis au jour un habitat rural dans lequel est apparue une très faible quantité de céramiques d'Iznik et de lustre iranien safavide alors que les fincan de Kütahya et les tasses de porcelaine «bleu et blanc» du XVIII ${ }^{\mathrm{e}}$ siècle sont beaucoup plus nombreuses ${ }^{73}$. En Grèce aussi, il arrive que des fincan apparaissent dans des contextes modestes comme à Archondiki-le-Haut, un village de Béotie apparemment occupé aux $\mathrm{XVII}^{\mathrm{e}}$-XIX ${ }^{\mathrm{e}}$ siècles, dans lequel une seule et unique tasse de Kütahya a été retrouvée ${ }^{74}$. Ainsi qu'en attestent les fouilles, des tasses d'origines diverses ont été trouvées en petite quantité en contexte rural. Leur présence en ces lieux est peut-être liée à une pratique fréquente chez les voyageurs orientaux qui consistait, aux $\mathrm{XVII}^{\mathrm{e}}$ et $\mathrm{XVIII}^{\mathrm{e}}$ siècles, à serrer à l'intérieur de boîtes cylindriques en cuivre ou dans des étuis de maroquin «des tasses de porcelaines pour offrir à boire le cavet à ceux qui rendent visite suivant la coutume des levantins ${ }^{75}$. Les tasses brisées en chemin étaient abandonnées.

\section{DISCORDANCE ENTRE SOURCES ÉCRITES ET DONNÉES MATÉRIELLES}

À la lecture de ce qui précède plusieurs remarques s'imposent. Le nombre de fincan mis au jour est très faible par rapport aux chiffres donnés dans les textes - rappelons par exemple que dans les inventaires des cafés du Caire, au XVIII ${ }^{\mathrm{e}}$ siècle, le nombre de tasses varie entre 30 et 210. À la citadelle de Damas, les $1600 \mathrm{~m}^{2}$ fouillés ont livré 7844 fragments d'époque ottomane dont seulement 94 tasses (46 de Kütahya,

71 T. Sмiтh, Remarks upon the Manners, Religion, and Government of the Turks. Together with a Survey of the Seven Churches of Asia, Londres, 1678, p. 235-239; Evliya ÇELEBI, Seyahatname, Istanbul, 1935, vol. 9, p. 55.

72 J. Aegidius van Egmont, J. Heyman, Travels through Part of Europe, Asia Minor, the Islands of the Archipelago, Syria, Palestine, Egypt, Mount Sinai, etc., Londres, 1759, p. 148-150.

73 H. CRAnE, «Some Archaeological Notes on Turkish Sardis », Muqarnas 4, 1987, p. $43-58$.

74 J. Vroom, «Coffee and Archaeology. A Note on a Kütahya Ware Find in Beotia, Greece», Pharos IV, 1996, p. 5-19.

75 La Boullaye-Le-Gouz, Les voyages et observations du sieur de La Boullaye-LeGouz, gentilhomme angevin, Paris, 1653, p. 60-61 ; H. DESMET-GrEGOIRE, 1989, fig. 78 ; eadem, "Origine et évolution du café à Marseille aux XVII et XVIII siècles », Provence Historique, XXXVIII, fasc. 151, janvier-mars 1988, p. 78; H. AMOURIC, Fl. RicheZ, L. VAllaurI, Vingt mille pots sous les mers, Aix-en-Provence, 1999, p. 161 ; C. EstABLET, J.-P. PASQUAL, 2001, p. 147. 
28 de Chine, 20 de Damas, 2 de Meissen et une d'Iran). De façon plus générale, les quantités mises au jour sur les sites d'Anatolie et du Bilâdal-Châm sont toujours faibles. L'interprétation des textes a souvent conduit à la hiérarchisation des objets établissant que les productions locales et les objets importés ne s'adressaient pas à la même clientèle, porcelaine et faïence de très haute qualité n'étant répandues que dans les classes privilégiées, en majorité urbaines. Or la comparaison des prix des tasses mentionnés dans les inventaires montre qu'il est bien difficile de distinguer les objets de luxe des autres (Tableau 1).

\begin{tabular}{|c|c|}
\hline Type de tasse & Prix à l'unité \\
\hline \multicolumn{2}{|c|}{ Istanbul, début XVII ${ }^{\mathrm{e}} \mathrm{s}$. } \\
\hline $\begin{array}{l}\text { altunli (dorées) } \\
\text { sade (simples) }\end{array}$ & $\begin{array}{l}5 \text { akçe } \\
2 \text { akçe }\end{array}$ \\
\hline \multicolumn{2}{|c|}{ Ankara, XVII' s. } \\
\hline $\begin{array}{c}\text { acemi } \\
\text { Kabe fincani } \\
\text { Fağfuri }\end{array}$ & $\begin{array}{l}1 \text { esedi guruş } \\
0,2 \text { esedi gurus } \\
0,1 \text { esedi guruş }\end{array}$ \\
\hline \multicolumn{2}{|c|}{ Le Caire, XVII ${ }^{\mathrm{e}}$ s. } \\
\hline Tasses de Hays & 0,8 paras \\
\hline \multicolumn{2}{|c|}{ Le Caire, XVIII ${ }^{\mathrm{e}}$ s. } \\
\hline fincân sîni & de 15 à 20 paras \\
\hline \multicolumn{2}{|c|}{ Damas, début XVIII ${ }^{\mathrm{e}} \mathrm{s}$. } \\
\hline $\begin{array}{l}\text { gümüsslü } \\
\text { may } \\
\text { djadid } \\
\text { Farğfûri } \\
\text { sînî } \\
\text { simple } \\
\text { 'adjami } \\
\text { 'asali }\end{array}$ & $\begin{array}{c}3,5 \text { piastres } \\
0,5 \text { piastres } \\
0,37 \text { piastres } \\
0,14 \text { piastres } \\
0,11 \text { piastres } \\
0,10 \text { piastres } \\
0,09 \text { piastres } \\
0,07 \text { piastres }\end{array}$ \\
\hline
\end{tabular}

TABlEAU 1. Valeurs des tasses livrées par les textes

À Ankara, par exemple, au XVII ${ }^{\mathrm{e}}$ siècle, une tasse chinoise était dix fois moins chère qu'un fincan 'acemi. À Damas, au XVIII ${ }^{\mathrm{e}}$ siècle, les porcelaines de Chine avaient un prix moyen, tandis que - si on isole la tasse gümüşlü, peut-être en argent — la tasse bleue (may), la plus précieuse, est sept fois plus chère que le fincan 'asali. Il est donc malaisé 
d'établir une hiérarchie de ces objets sur la base de leur valeur estimée, d'autant que cette valeur a pu varier d'un siècle à un autre et d'un lieu à un autre. Compte tenu des volumes assez importants de tasses chinoises, de fincan de Kütahya et de porcelaine de Meissen (2 000 tasses commandées en 1732 à la manufacture par un négociant turc), il est permis de penser que ces objets, au XVIII ${ }^{\mathrm{e}}$ siècle, étaient relativement bon marché. Produits en grand nombre, bien diffusés, ils étaient accessibles aussi bien à un tenancier de café du Caire, qu'à certains marchands du Caire, d'Ankara ou de Bursa, ainsi qu'aux pèlerins de retour de La Mecque. De la même façon, la présence de porcelaine chinoise, de céramique de Kütahya et de porcelaine de Saxe à Sardes, à Aphrodisias ou dans des habitats modestes de Grèce centrale ne va pas dans le sens d'objets coûteux réservés à une élite fortunée. Les textes et les données matérielles militent en faveur de produits assez répandus dans toutes sortes de milieux sans qu'il soit réellement possible de distinguer les tasses respectivement utilisées par l'élite et par le commun. D'autant qu'on ne trouve pas, sur les sites, de trace archéologique de substituts bon marché aux tasses chinoises et aux productions anatoliennes - des petits récipients de mêmes formes simplement fabriqués en terre cuite et couverts d'une glaçure plombifère par exemple. Les tasses communes, parfois évoquées dans les sources écrites, font défaut sur le terrain même dans des contextes modestes dans lesquels apparaissent par ailleurs des fincan importés. Qu'en est-il donc de la «rusticité des objets destinés au peuple» évoquée par les historiens? Leur absence dans les fouilles signifie-t-elle que ces tasses sans valeur ont toutes disparu? S'il est possible que les tasses de bois tourné n'aient pas résisté aux dommages du temps et que les gobelets fabriqués en laiton ou en cuivre occasionnellement étamé, comme ceux exposés au Palais Azem à Damas (pl. V : 2-4), aient été refondus, il est plus difficile d'expliquer la disparition de tasses communes en terre. En fait, l'élément discriminant entre pauvres et riches était peut-être le zarf et non la tasse. Rappelons que ces petits godets hémisphériques de terre étaient dépourvus d'anse, aussi, afin que le buveur ne se brûle pas les doigts, ils pouvaient être placés dans des zarf, c'est-à-dire des supports de métal ouvragé, en cuivre, en laiton, en argent ou en vermeil de la forme d'un coquetier, parfois munis d'une anse et montés sur un petit pied facilitant la préhension de l'ensemble. Dans les cafés publics, selon Chabrol «Les tasses sont posées dans de petits gobelets en cuivre, qui ressemblent à ces vases de faïence connus parmi nous sous le nom de coquetiers, et que les Arabes nomment $z a r f{ }^{76}$. Chez les puissants, ces supports pouvaient être très ornés. Evliya Çelebi, reçu par le Khan de Bitlis, dans les années 1650, fut émerveillé par le service de ce dignitaire car «On servit dans des tasses incrustées de pierres précieuses du café du Yémen

76 Chabrol, vol. XVIII, Paris, 1826.

77 Evliya Çelebi cité par H. Desmet-Gregoire, 1989, p. 81. 
parfumé d'ambre ${ }^{77}$. Plus tard, Des Planches rapporte que le propriétaire d'un riche magasin de Damas «n'oublia pas de nous faire apporter selon les usages orientaux l'inévitable moka qui nous fut servi dans des tasses précieuses reposant elles-mêmes dans des vases à jour tout ciselés d'or fin ${ }^{78}$. Ces coquetiers ouvragés faisaient tellement partie du service à café que la Manufacture de Sèvres, pour répondre aux goûts des Turcs, fabriqua en 1792, «un porte-tasse en or émaillé en blanc garni de diamants » et en 1861 et 1873 , créa des zarfs réticulés en porcelaine dure, rehaussée d'or ${ }^{79}$. Il semble que ces objets étaient essentiellement utilisés par l'élite ottomane et par ceux qui souhaitaient s'en approcher par les manières. Au-delà de leur fonction utilitaire, les zarfs fabriqués avec des matériaux précieux et incrustés de pierres fines étaient des moyens pour l'hôte de révéler son opulence et de souligner l'importance de ses invités. Mais ces enveloppes de métal masquaient presque totalement les tasses de porcelaine de Chine ou de Saxe dont on n'apercevait plus que le bord sur lequel poser les lèvres. Dans ce système de luxe ostentatoire, on comprend mal que, si les Ottomans attachaient beaucoup de valeur aux tasses, ils les dissimulaient sous une enveloppe de métal.

Comme le rapportent souvent les textes, dans l'Empire ottoman, il n'était pas rare de trouver des cafés qui ne comportaient ni murs, ni toits pour les délimiter, simplement un espace marqué par quelques tables et des bancs en plein air. Les repères ne résidaient-ils pas alors simplement dans les principaux objets utilisés en ces lieux, dont le fincan, morphologiquement né en Chine pour servir de contenant au vin, au thé et à l'alcool de riz, puis adopté et copié dans les ateliers arabes du Yémen et dans les centres de productions ottomans d'Anatolie, d'Egypte et du Bilâd al-Châm, afin de servir de récipient pour boire le café. Elément incontournable de la culture ottomane, utilisé dans les sphères privées et publiques, cet objet très présent dans les sources écrites est plus rare sur le terrain bien qu'il fut très populaire. Lorsque la porcelaine européenne atteignit sa plénitude aux XVIII ${ }^{\mathrm{e}}$ et $\mathrm{XIX}^{\mathrm{e}}$ siècles, elle eut la faveur des Orientaux qui ne renoncèrent pas pour autant aux modèles auxquels ils étaient attachés. Les inventaires des grandes manufactures de Sèvres ou de Meissen témoignent bien de la suprématie du fincan. Il y est en effet question de «tasses pour le turc» ou «tasses pour zarph». Les faïenciers de Marseille surent également s'adapter à leur clientèle turque. Le terme fincan faisait partie, dès la première moitié du $\mathrm{XVIII}^{\mathrm{e}}$ siècle, de leur vocabulaire et, en 1806, un procès verbal de la Chambre de Commerce de cette ville fait encore état d'échantillons de pièces «remarquables par l'attention qu'a eue le fabriquant de les choisir adaptées au

78 Des Planches, Orient. Excursions, descriptions, récits, études, Marseille, 1900, p. 141 .

79 H. Desmet-Gregoire, 1989, p. 76, 93. 
goût des Turcs... ${ }^{80}$. Les artisans avaient sans doute eu pour modèles les tasses importées de Kütahya, retrouvées sur divers sites de Provence et mentionnées souvent dans les inventaires mobiliers des personnes de qualité comme «fingeans pour le caffé » ${ }^{81}$ (pl. V: 5). Le fincan, adopté par certains Provençaux au XVIII siècle et copié à Marseille, Moustier et Gênes, allait côtoyer un temps en Europe des tasses à café de types différents mais ceci est une autre histoire.

${ }^{80}$ H. Desmet-Gregoire, 1989, p. 82. Sur les productions des ateliers de Marseille et de Moustier, voir H. Amouric, Fl. Richez, L. Vallauri, 1999, p. 163-167.

81 H. Amouric, Fl. RicheZ, L. VAllauri, 1999, p. 160-167. 


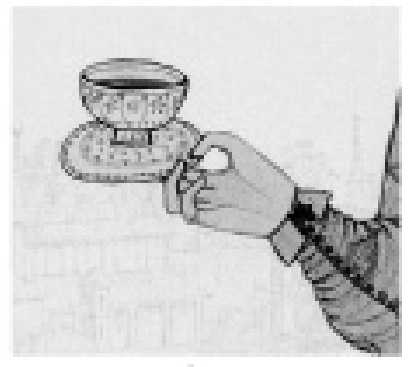

1

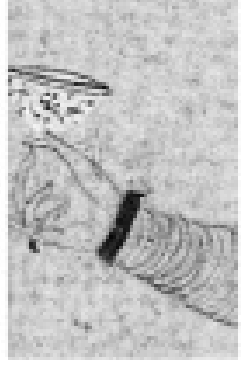

2

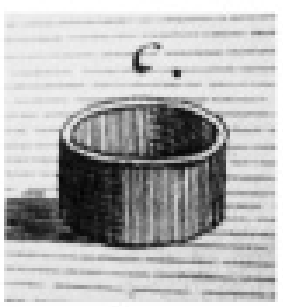

5

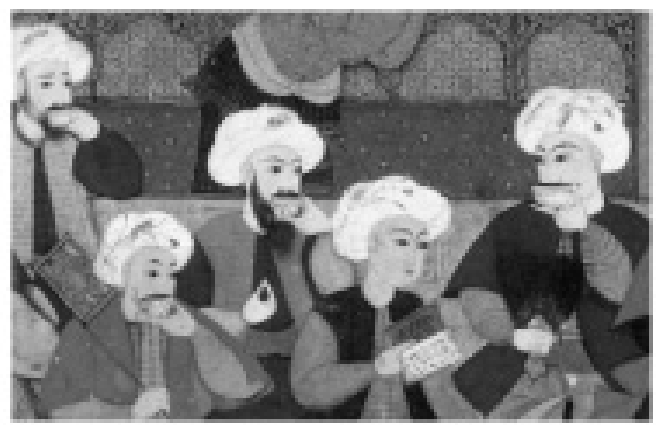

3

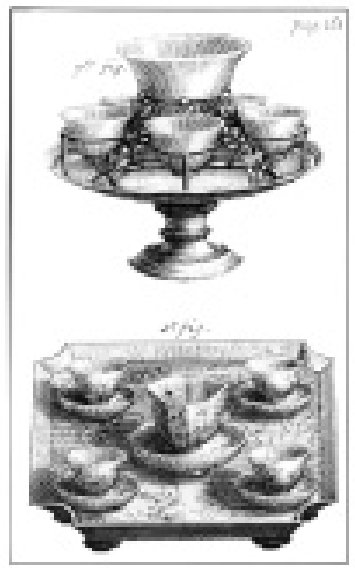

4

Pl. I : Tasses en porcelaine de Chine utilisées à la cour ottomane (1 et 2) (d'aprís Chinese Treasures, 2001) et dans les cafés de la capitale (3) (d'aprés R.S. Hattox, 1988) ; services de tasses ottomanes vus par les Occidentaux (4) (d'aprés N. de Blegny, 1687) ; tasse de "pierre de potier" du Yémen (5) (d'aprés C. Niebuhr, 1779) 

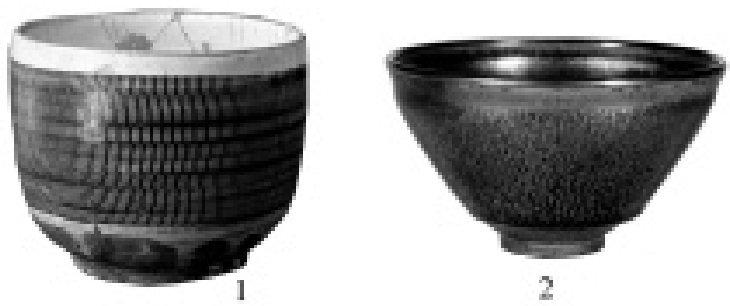

2
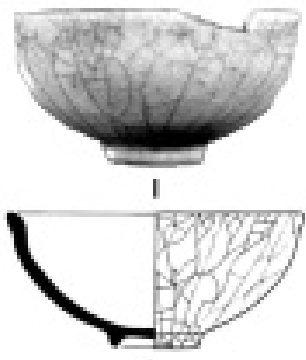

3
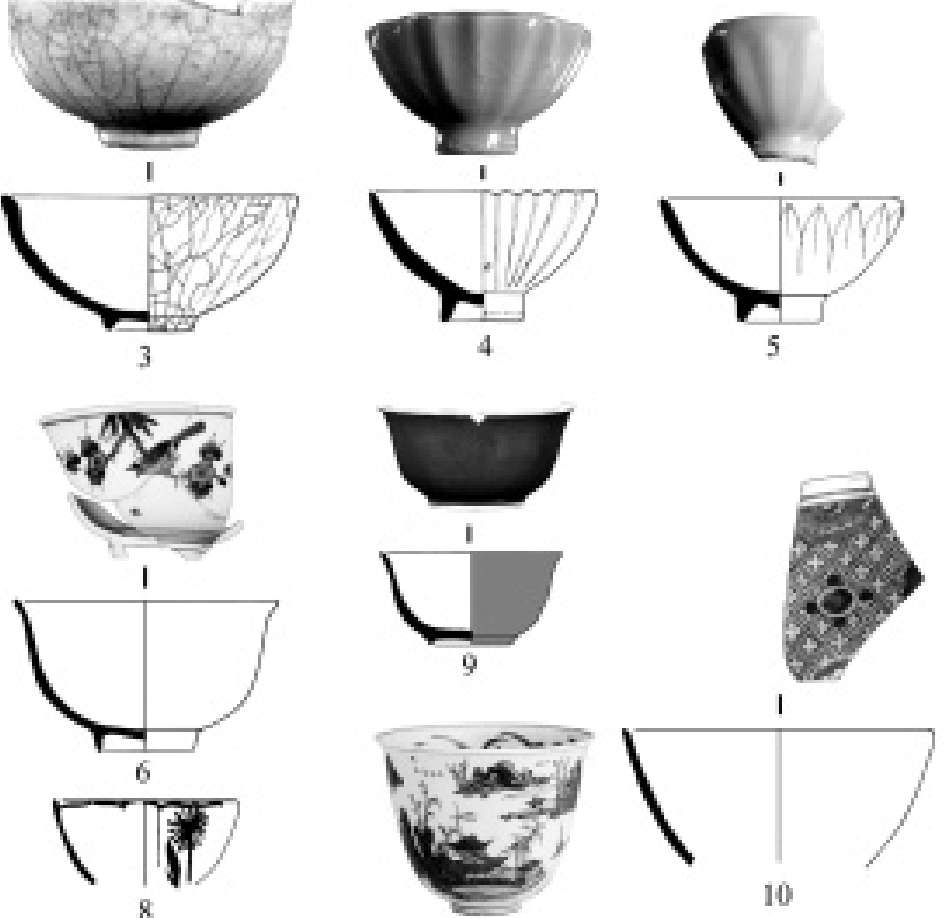

7

10

Pl. II : Grès Jian du Fujian (1, 2) (d'aprls J. Ayers ef al., 1984) ; céladon Guan de Hangzou (3) et céladons de Longquan (4-5), d'époque Song ; porcelaines. "bleu et blanc" d'époque Ming $(6,7)$; porcelaines "bleu et blanc" $(8)$; porcelaine Café-au-lait (9) et imari chinois (10) d'époque Qing. Ech. 1:3 
ÉLÉMENTS POUR UNE BIOGRAPHIE DES TASSES À CAFÉ 317

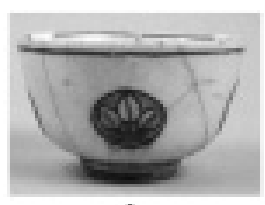

1

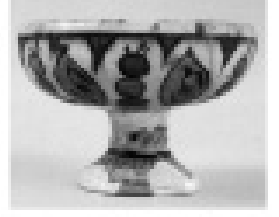

2

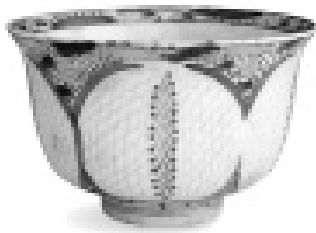

3

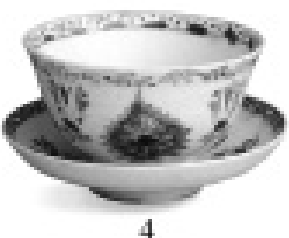

4
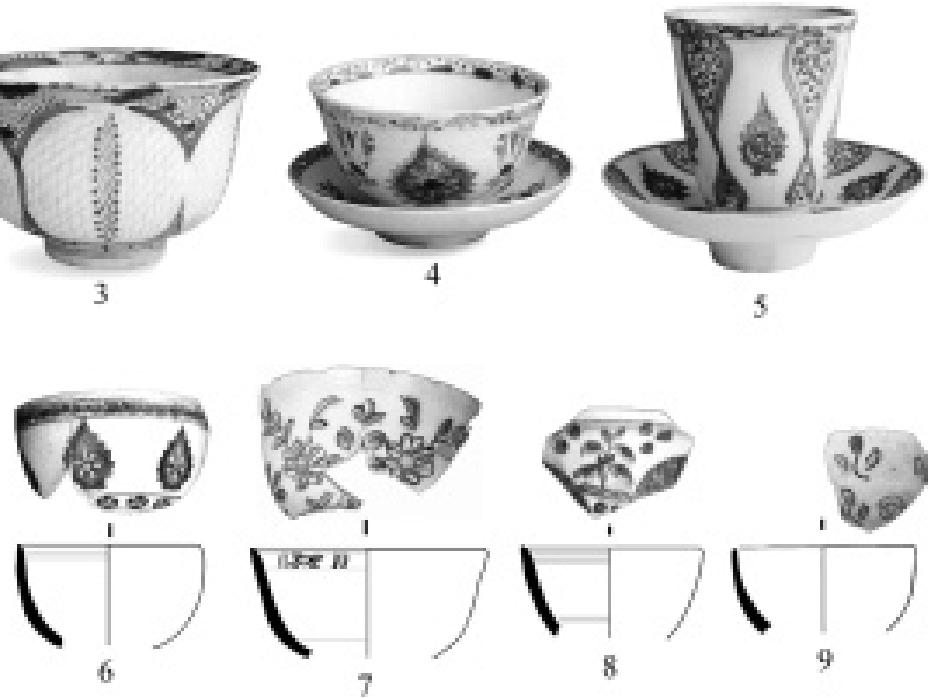

8
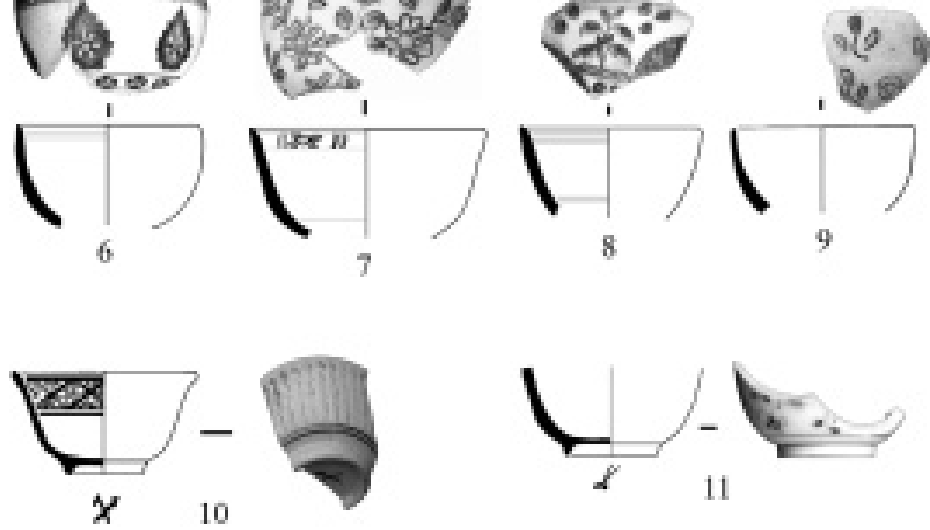

Pl. III : Fincan d'Iznik (1-2) (d'apre's N. Atasoy, J. Raby, 1990), de Kütahya (3-9) (d'après L. Soustiel, 2000) et tasses de Meissen (10, 11). Ech. 1:3 
VÉRONIQUE FRANÇOIS

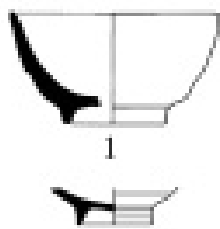

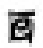

2
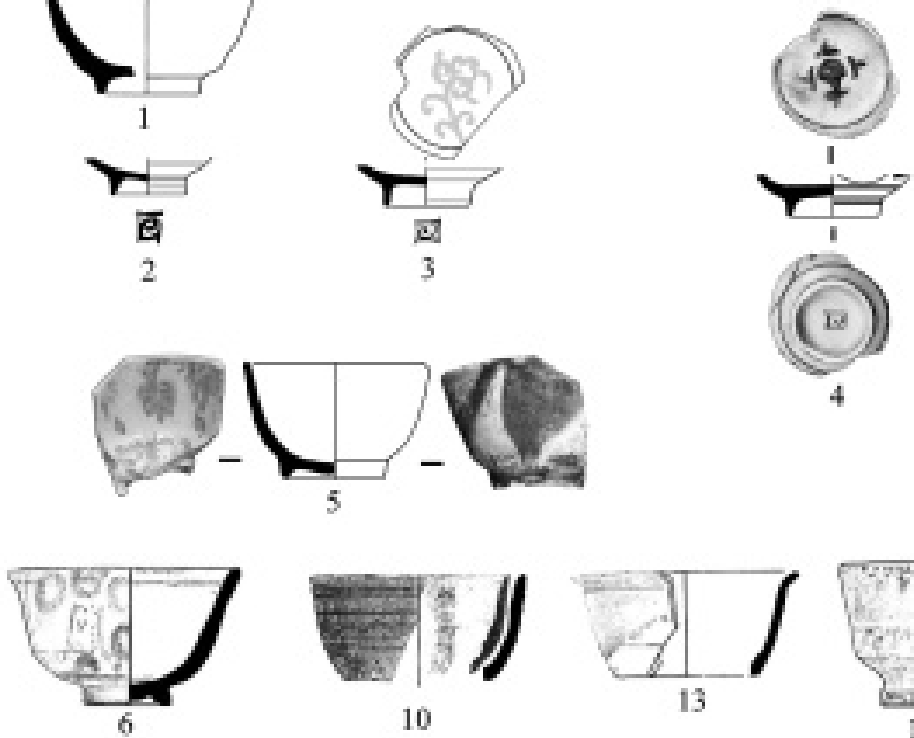

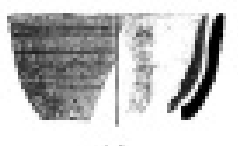

10

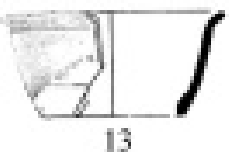

13

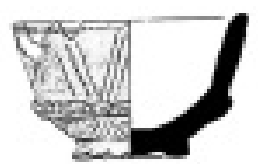

14

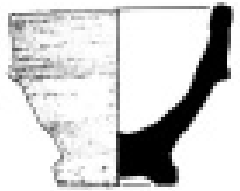

12

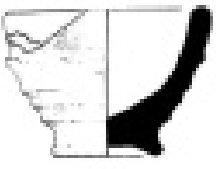

15
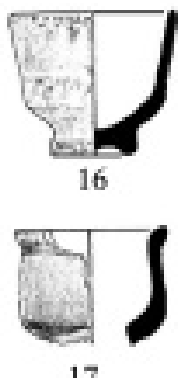

17

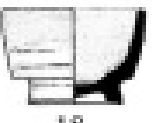

18

Pl. IV : Tasses çîi produites à Damas (1-4), tasses 'ajami de Perse safavide (5) et tasses des ateliers de Hays au Yémen (đaprès E.J. Keall, 2001) (6-19). Ech. 1:3 

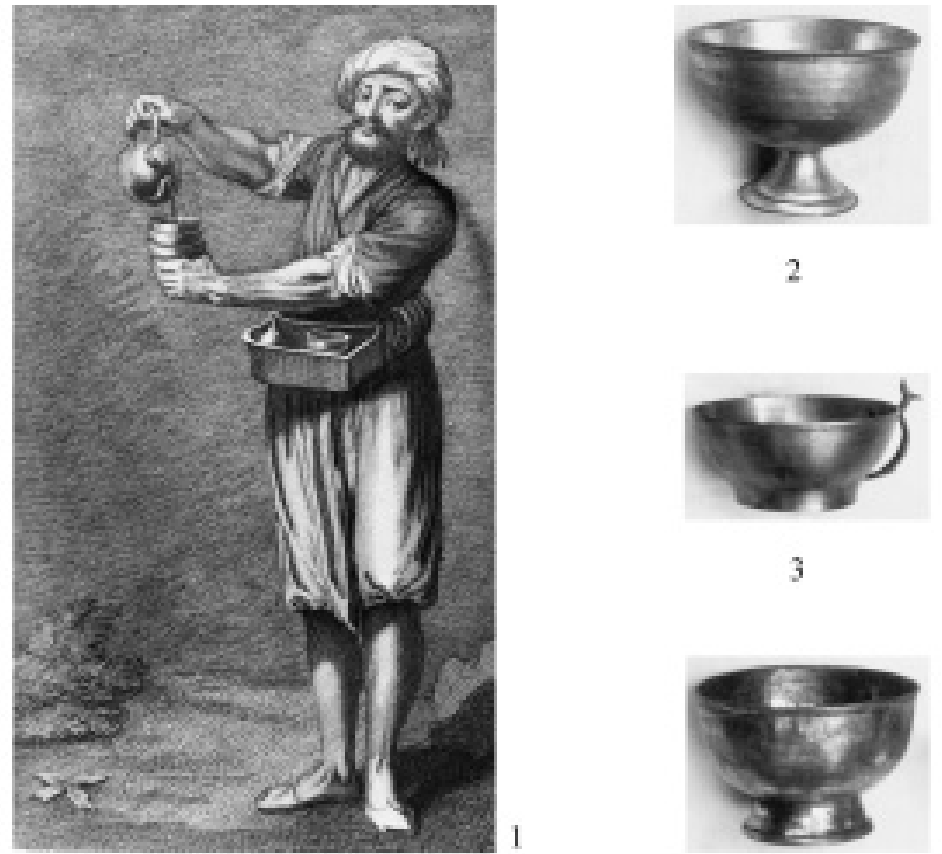

2

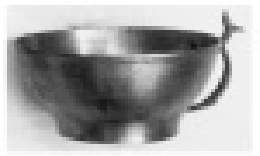

3

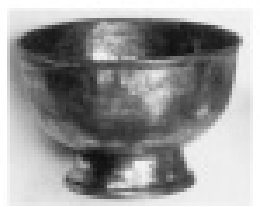

4

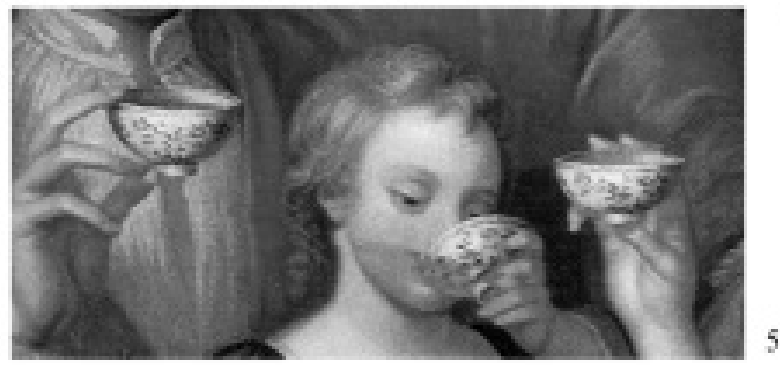

5

Pl. V : Vendeur ambulant de café à Istanbul (1) (d'après J. B. van Moor, 1714) ; tasses de laiton ou de cuivre étamé à Damas (2-4) (d'après C. Establet, J.P. Pascual, 2003) ; fingeans utilisés en Europe au XVIIIe siècle (5) 
Véronique FrançOIS, Éléments pour une biographie des tasses à café dans l'Empire ottoman

Si les travaux érudits ne manquent pas sur le thème du café, rares sont les auteurs qui se sont attardés sur la tasse à café, à l'exception de H. Desmet-Grégoire qui lui a consacré un chapitre entier dans sa monographie sur Les objets $d u$ café, examinés du point de vue de l'anthropologue. Aussi complète que soit son approche, elle s'appuie sur d'abondantes sources écrites et sur les fonds des musées des arts et traditions populaires mais ne prend pas en compte la réalité matérielle livrée par le terrain archéologique. Or cette dernière est différente, voire même en opposition avec ce que les textes rapportent sur la façon de boire le café; c'est ce que montrera cette étude. À partir de la confrontation des sources écrites et des données matérielles, cet article propose une histoire de la tasse à café dans les mondes arabe et turco-ottoman.

Véronique FrançoIs, Elements for a biography of coffee cups in the Ottoman Empire

Although we are not short of well informed studies concerning coffee, very few authors have dealt with the coffee cup, except H. Desmet-Grégoire who devoted a whole chapter on the subject in her monography, Les objets du café, studied from an anthropologist point of view. Yet, however comprehensive, her approach relies on many written sources and on Art and Popular Tradition Museum collections, but does not take into account the actual reality offered by archaeology. As a matter of fact, the latter is different, even in contradiction with what the written texts tell us about the drinking coffee ways. This is the subject of this study. Starting with a comparison of written sources and actual archaeological data we propose a history of the coffee cup in Arab and TurcoOttoman worlds. 